17) Marcus, Y. and Z. Kolarik: J. Inorg. Nucl. Chem., 38, 1069 (1976).

18) McDowell, W. J. and C. F. Coleman: J. Inorg. Nucl. Chem., 27, 1117 (1965).

19) Murayama, K.: Shin-Kinzokukogyo, No. 12, 1 (1975).

20) Nakashio, F., K. Kondo, A. Murakami and Y. Akiyoshi: $J$. Chem. Eng. Japan, 15, 274 (1982)

21) Partridge, J. A. and R. C. Jensen: J. Inorg. Nucl. Chem., 31, 2587 (1969).

22) Peppard, J. P., G. W. Mason and D. F. Peppard: J. Inorg. Nucl. Chem., 22, 285 (1961).
23) Peppard, D. F., M. N. Namboodri and G. W. Mason: $J$. Inorg. Nucl. Chem., 24, 979 (1962).

24) Preston, J. S., J. Inorg. Nucl. Chem., 37, 1235 (1975).

25) Ritcey, J. M., A. W. Ashbrook and B. H. Lucas: CIM Bulletin, 111 (1975).

27) Smelov, V. S., V. P. Lanin, Z. A. Smyk and V. V. Chubukov: Radiokhimiya, 14, 352 (1972).

28) Uchida, S., K. Takahashi and H. Takeuchi: Proceedings of Symp. of Solv. Ext. of Metals, Hamamatsu, Dec. 11-12, paper 1A-03 (1981).

29) Warshawsky, A.: Miner. Sci. Eng., 5, 36 (1973).

\title{
KINETIC STUDY OF OXIDATION OF PELLETED ZINC SULFIDE POWDER HAVING GRAIN SIZE DISTRIBUTION
}

\author{
Shoichi KIMURA, YoshikazU TAKAGI, Setsuji TONE \\ AND TSUTAO OTAKE \\ Department of Chemical Engineering, Faculty of Engineering Science, \\ Osaka University, Toyonaka 560
}

\begin{abstract}
Oxidation of porous zinc sulfide pellets, composed of fine grains having log-normal size distributions, was carried out using a differential flow reactor under oxygen partial pressure of $1-16 \mathrm{kPa}$ and temperature of $953-1113 \mathrm{~K}$.

The overall conversion rate data were represented by a simple power law rate expression. The reaction order with respect to the solid reactant varied with the standard deviation of the grain size distribution. This variation agreed well with the theoretical prediction by the grain model accounting for grain size distribution. The reaction order with respect to the gaseous reactant was $1 / 2$.
\end{abstract}

The surface rate constant was then found to be correlated in an Arrhenius equation.

$$
k^{\prime}=5.56 \times 10^{8} \mathrm{e}^{-244 / R T} \quad\left(\mathrm{~mol}^{1 / 2} / \mathrm{m}^{1 / 2} \cdot \mathrm{s}\right)
$$

The rate constant agreed well with that for oxidation of sphalerite single crystals by other workers as well as with the rate constant reproduced from the data in the literature on oxidation of porous pelleted zinc sulfide.

\section{Introduction}

Gas-solid reactions involving a porous solid are numerous and industrially of great importance. For the interpretation and application of rate data observed in a variety of gas-solid reactions a number of kinetic models have been proposed.

It has been known that the kinetics by which the reaction proceeds depends entirely on the structure of the solid phase. The kinetic model describing the data is therefore required to reflect somehow the actual structure of the solid. In this light, the grain mod$\mathrm{el}^{16,18)}$ may be noteworthy for its simple but practical microscopic structural representation. This model assumes that the porous solid is an ensemble of grains of uniform size. A gaseous reactant diffuses through pores between grains and reacts on the unreacted core

Received August 9, 1982. Correspondence concerning this article should be addressed to S. Kimura. formed in each grain.

The grain model is expected to be especially applicable when a pellet involved in the reaction is molded using powdered solid reactant by hand rolling, compression, sintering, etc. In the usual situation, however, the grains of the powdered reactant are considered not to be uniform in size but rather to have a size distribution. In such cases the model assuming uniform grain size may not be properly applied. Several workers ${ }^{1,10,15)}$ have predicted by numerical calculation the influence exerted upon the gross behavior by the grain size distribution. Although they pointed out the differences from the model assuming uniform grains, it was not very simple to apply their results to practical situations.

In this work the oxidation of pelletized zinc sulfide powder is investigated in detail. The rate data are analyzed on the basis of a simple rate equation derived from a grain model accounting for the grain 
size distribution, ${ }^{8)}$ and the rate constant is found to be correlated in an Arrhenius equation. The rate data in the literature are also subjected to interpretation by this kinetic model for comparison with the results of this work.

\section{Experimental}

\subsection{Experimental apparatus and procedures}

A differential reactor, a quartz tube of $10 \mathrm{~mm}$ inner diameter, was used to measure the rate of oxidation reaction. Oxygen concentration in the reactant gas was adjusted by controlling flow rates of air and inert nitrogen. The flow rate and oxygen content of the reactant gas were maintained at levels where both gasfilm resistance and intraparticle temperature rise due to heat of reaction were negligibly small.

Pelleted sample of powdered zinc sulfide was sandwiched between quartz wool in the reactor tube and heated to a prescribed reaction temperature in a stream of inert nitrogen. The sample was then contacted with reactant gas by switching the streamlines connected to the reactor.

The progress of oxidation was followed by detecting $\mathrm{SO}_{2}$ gas produced according to ${ }^{2-4,6,14)}$

$$
\mathrm{ZnS}+\frac{3}{2} \mathrm{O}_{2} \rightarrow \mathrm{ZnO}+\mathrm{SO}_{2}
$$

The concentration of $\mathrm{SO}_{2}$ in the effluent gas from the reactor was measured by an IR stream-line $\mathrm{SO}_{2}$ analyzer.

The extent of conversion from zinc sulfide to zinc oxide was calculated by

$$
\bar{X}_{B}=\frac{\text { cumulative amount of } \mathrm{SO}_{2} \text { produced at any time }}{\text { total moles of } \mathrm{SO}_{2} \text { produced by the end of reaction }}
$$

The cumulative amount of $\mathrm{SO}_{2}$ was determined by graphical integration of production rate curves of $\mathrm{SO}_{2}$. The end of reaction was confirmed by detecting no $\mathrm{SO}_{2}$ in the effluent gas. The total moles of product $\mathrm{SO}_{2}$ gas obtained by graphical integration agreed well with the sulfur content of the sample within $\pm 1 \%$ error.

\subsection{Materials}

1.2.1 Preparation of zinc sulfide pellets The original powdered zinc sulfide $(99.9 \%$ crystalline $\mathrm{ZnS}$ : Mituwa Pure Chemicals) was screened once, using a $20 \mu \mathrm{m}$ microsieve by way of precaution to remove peculiarly large grains. The powder was then screened at need by sets of microsieves to adjust the grain size in several ranks. For this purpose, combinations of microsieves of $5,8,10,12.5,15 \mu \mathrm{m}$ were used.

The screened powder was blended with a small amount of ethanol to make a thick paste so that grains cohere, followed by the evaporation of ethanol at room temperature. The cake of solid obtained was cut into pellets of about $1 \mathrm{~mm}$ in size. The edges of

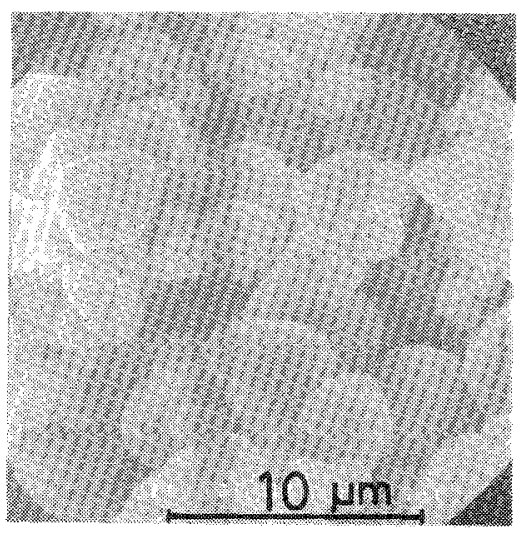

Fig. 1. SEM photograph of $\mathrm{ZnS}$ grains constituting sample pellets.

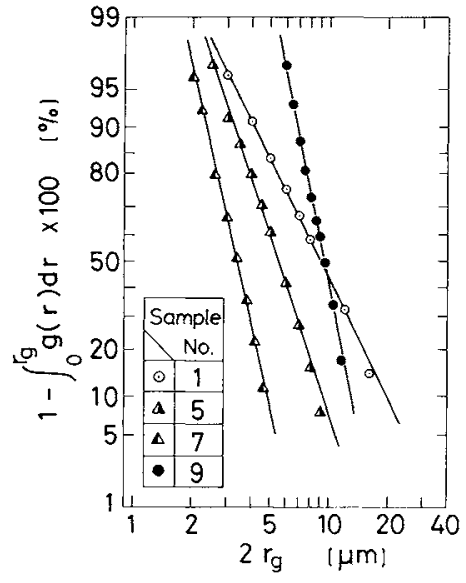

Fig. 2. Test of grain size distribution on log-normal probability chart.

pellets were rounded off by rolling in a mortar so that the pellets became spherical. Size of sample pellets was adjusted in the rage $0.59-0.71 \mathrm{~mm}$, for which size the intraparticle diffusion resistance was confirmed by preliminary experiments not to influence the oxidation rate in the temperature range investigated. For each oxidation run, about 10 sample pellets were used, being sandwiched in scattered fashion between quartz wool in the reactor tube.

Figure 1 shows a SEM photograph of a halved sample pellet prepared in the manner described above. The pellet is very porous, being composed of fine nonporous grains which are nearly spherical and nonuniform in size. There is neither sintering nor interconnection observed between grains.

1.2.2 Size distribution of powdered zinc sulfide The size distribution of powdered zinc sulfide constituting sample pellets was measured by the phototransmission method. The cumulative weight fraction of grains greater than given size was observed by a centrifugal particle size analyzer (Shimadzu CP50).

Figure 2 illustrates the distribution of the zinc sulfide powder on a log-normal probability chart, taking a few cases as examples. The cumulative 
Table 1. Physical properties of zinc sulfide grains constituting sample pellets

\begin{tabular}{|c|c|c|}
\hline $\begin{array}{c}\text { Sample } \\
\text { No. } \\
{[-]}\end{array}$ & $\begin{array}{c}\text { Mean grain } \\
\text { diameter } \\
2 \bar{r}_{g} \times 10^{6} \\
{[\dot{\mathrm{m}}]}\end{array}$ & $\begin{array}{c}\text { Standard } \\
\text { deviation } \\
\sigma \\
{[-]}\end{array}$ \\
\hline 1 & 8.9 & 0.59 \\
\hline 2 & 6.4 & 0.48 \\
\hline 3 & 10.3 & 0.45 \\
\hline 4 & 5.2 & 0.43 \\
\hline 5 & 5.5 & 0.40 \\
\hline 6 & 6.6 & 0.36 \\
\hline 7 & 3.4 & 0.28 \\
\hline 8 & 8.7 & 0.26 \\
\hline 9 & 9.5 & 0.23 \\
\hline
\end{tabular}

weight fractions of grains have linear relationships with grain size on this probability chart. The straight lines indicate that the zinc sulfide grains have a lognormal size distribution on the basis of weight, the density function of which is given by

$$
g\left(r_{g}\right)=\frac{1}{\sqrt{2 \pi} \sigma} \frac{1}{r_{g}} \exp \left[-\left(\ln \frac{r_{g}}{\bar{r}_{g}}\right)^{2} / 2 \sigma^{2}\right]
$$

The mean grain radius $\bar{r}_{g}$ and the standard deviation $\sigma$ determined on the probability chart are shown for each sample in Table 1. The original grains have a widely spreading distribution while the screened grains have distributions in a narrower range.

\section{Rate Equation by Grain Model with Size Distri- bution}

The porous pellets used in this work are composed of fine spherical grains, which suggests that the grain model ${ }^{16,18)}$ is applicable. One may understand, however, that the grain model should be modified so that grain size distribution is taken into consideration.

Suppose that the following general gas-solid reaction is taking place in each individual grain forming an unreacted core.

$$
\mathrm{A} \text { (gas) }+b \mathrm{~B} \text { (solid) } \longrightarrow \text { products }
$$

When the resistance to product layer diffusion in each grain is negligible, the progress of reaction in the grain is represented by ${ }^{9}$ )

$$
\frac{t}{\tau}=1-\left(1-X_{B}\right)^{1 / 3} ; \quad 0 \leq t \leq \tau
$$

where $X_{B}$ signifies the extent of conversion of the grain at any time. The time required for complete conversion $\tau$ of a grain having radius $r_{g}$ is given by ${ }^{7,9)}$

$$
\tau=\frac{r_{g}}{b k^{\prime} C_{A}{ }^{n} v_{B}}
$$

where $k^{\prime}$ is the surface reaction rate constant and $v_{B}$ is the molar volume of B. $C_{A}$ is the concentration of $\mathrm{A}$ in the environment surrounding the grain, and $n$ is the reaction order with respect to the gaseous reactant.

When the resistance to diffusion through pores between grains in the pellet is also negligible, the concentration of $\mathrm{A}$ is uniform throughout the pellet. Then, using a density function $f(\tau) d \tau$, the overall conversion $\bar{X}_{B}$ of the pellet at any time is given by Eq. (4).

$$
\bar{X}_{B}=1-\int_{t}^{\infty}\left(1-\frac{t}{\tau}\right)^{3} f(\tau) d \tau
$$

In Eq. (6), $f(\tau) d \tau$ gives the weight fraction of grains which are completely converted in the time $\tau$ to $\tau+d \tau$.

The derivative of Eq. (6) with respect to $t$ gives the overall conversion rate of the pellet, or

$$
\frac{d \bar{X}_{B}}{d t}=3 \int_{t}^{\infty} \frac{1}{\tau}\left(1-\frac{t}{\tau}\right)^{2} f(\tau) d \tau
$$

Since the complete conversion time $\tau$ is linearly correlated to grain size $r_{g}$ by Eq. (5), $g\left(r_{g}\right)$ is directly transformed into $f(\tau)$. Thus, with some manipulation, Eqs. (6) and (7) reduce to ${ }^{8)}$

$$
\begin{gathered}
\bar{X}_{B}=1-\frac{1}{\sqrt{2 \pi}} \int_{\ln (t / \bar{\tau}) / \sigma}^{\infty}\left(1-\frac{t}{\bar{\tau}} \mathrm{e}^{-\sigma \xi}\right)^{3} \\
\times \exp \left[-\frac{\xi^{2}}{2}\right] d \xi \\
\frac{d \bar{X}_{B}}{d t}=\frac{3 e^{\sigma^{2} / 2}}{\sqrt{2 \pi}} \int_{\ln (t / \bar{\tau}) / \sigma}^{\infty}\left(1-\frac{t}{\bar{\tau}} \mathrm{e}^{-\sigma \xi}\right)^{2} \\
\times \exp \left[-\frac{(\sigma+\xi)^{2}}{2}\right] d \xi
\end{gathered}
$$

where $\xi$ is defined by $\xi=\ln (\tau / \bar{\tau}) / \sigma$ and $\bar{\tau}$ is the mean of the complete conversion time.

When plotting $d \bar{X}_{B} / d t$ against $1-\bar{X}_{B}$ in $\log$-log scale, both of which are calculated for a given value of $\sigma$ by Eqs. (8) and (9), one may find that these two terms are approximated by a straight line for $\sigma \leq 1{ }^{8}$ ) Thus, in practical situations, one may correlate the overall conversion rate to the unconverted fraction by ${ }^{8)}$

$$
\frac{d \bar{X}_{B}}{d t}=\left(\frac{d \bar{X}_{B}}{d t}\right)_{t=0} \cdot\left(1-\bar{X}_{B}\right)^{m}
$$

With the aid of Eqs. (5) and (9), the initial conversion rate is given by

$$
\left(\frac{d \bar{X}_{B}}{d t}\right)_{t=0}=\frac{3 b k^{\prime} C_{A}{ }^{n} v_{B}}{\bar{r}_{g}} \exp \left(\sigma^{2} / 2\right)
$$

where $\bar{r}_{g}$ is the mean grain radius.

Equation (10) provides a power law rate expression. The reaction order $m$ with respect to the unconverted fraction $1-\bar{X}_{B}$ of solid varies with the standard deviation $\sigma$ of the grain size distribution, as shown by the solid line in Fig. 3. ${ }^{8)}$ When $\sigma$ goes to 0 , 


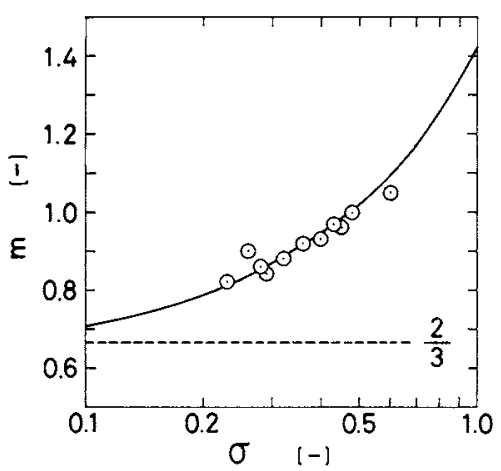

Fig. 3. Reaction order $m$ varying with standard deviation of grain size distribution.

that is, the size of grains is uniform, $m$ becomes $2 / 3$. In this case, Eq. (10) reduces to the expression obtained by the ordinary grain model.

Equation (10) suggests that plotting $\left(d \bar{X}_{B} / d t\right)$ observed at any extent of conversion $\bar{X}_{B}$ against $1-\bar{X}_{B}$ in $\log -\log$ scale yields a straight line having a slope $m$. The intercept of the straight line at $\bar{X}_{B}=0$ gives the initial conversion rate $\left(d \bar{X}_{B} / d t\right)_{t=0}$, which allows one to evaluate the rate constant $k^{\prime}$ by Eq. (11).

\section{Results and Analyses}

\subsection{Overall conversion rate}

Graphical differentiation of the fractional conversion of zinc sulfide varying with time provides the reaction rate of oxidation at any extent of conversion. The rate of conversion so obtained is plotted against the unconverted fraction in log-log scale in Fig. 4. As suggested by Eq. (10), satisfactory linear relationships are obtained except for the very initial stage of reaction. The discrepancy during the initial stage, which was neglected in the present work, is considered to be due to the existence of induction time.

The results for No. 5 sample, obtained under different reaction temperatures and oxygen concentrations, suggest that, although the magnitudes of $d \bar{X}_{B} / d t$ are different correspondingly, the slopes of the straight lines are identical independently of these reaction conditions. Thus, the difference of slopes between different samples, that is, Nos. 1, 5 and 9, may be considered to result from the difference in properties of the grains constituting the sample pellets.

\subsection{Test of data by the kinetic model}

Extrapolating the conversion rate to $\bar{X}_{B}=0$ provides the initial conversion rate $\left(d \bar{X}_{B} / d t\right)_{t=0}$. The order of reaction with respect to gaseous reactant may be evaluated by plotting $\left(d \bar{X}_{B} / d t\right)_{t=0}$ observed at a fixed temperature against oxygen concentration $C_{A}$ of the bulk reactant gas stream in $\log -\log$ scale. Thus, Fig. 5 indicates by the slope of the straight line that the reaction order $n$ yields $1 / 2$. This result agrees with that

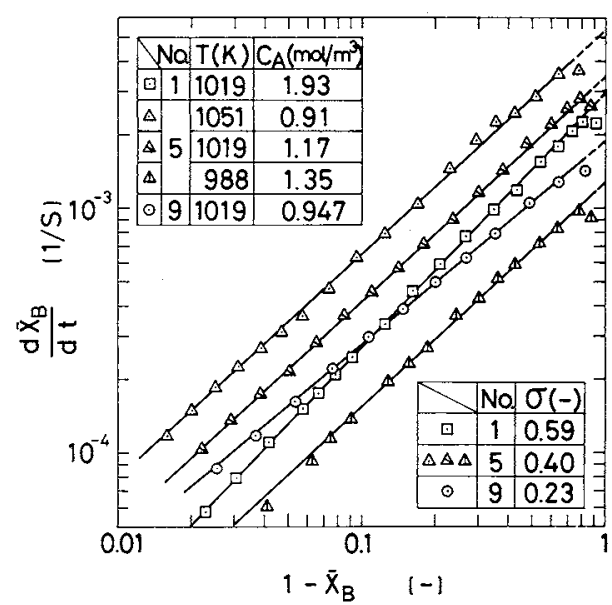

Fig. 4. Conversion rate plotted against unconverted fraction of solid based on Eq. (10).

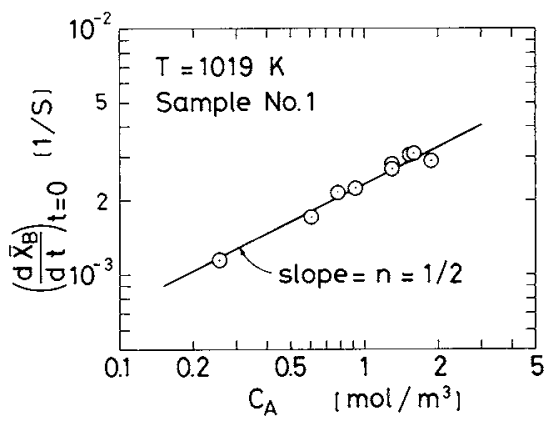

Fig. 5. Dependency of initial conversion rate on gaseous reactant concentrations.

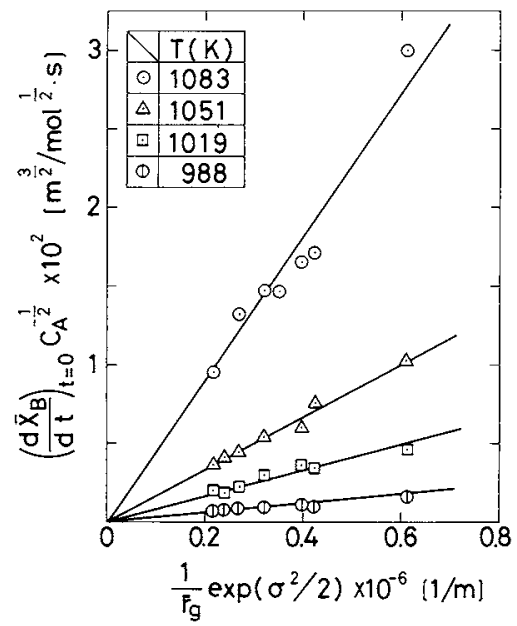

Fig. 6. Test of whether data satisfies requirements from kinetics based on Eq. (11).

observed by Cannon and Denbigh. ${ }^{2)}$

In Fig. 6 the initial conversion rate divided by the square root of $C_{A}$ is then plotted against $\exp \left[\sigma^{2} / 2\right] / \bar{r}_{g}$, based on Eq. (11). This plot critically tests whether the rate data satisfy the requirement concerning the properties of grains predicted by the presently proposed kinetics. The proportionality for each reaction temperature confirms that Eq. (11) holds.

The order of reaction $m$ with respect to solid 
Table 2. Reaction order $m$ obtained by slopes of straight line approximation based on Eq. (10)

\begin{tabular}{|c|c|c|c|c|c|}
\hline \multirow{2}{*}{$\begin{array}{c}\text { Sample } \\
\text { No. }\end{array}$} & \multicolumn{4}{|c|}{ Reaction temperature $T[\mathrm{~K}]$} & \multirow{2}{*}{$\begin{array}{c}\text { Av. } \\
\text { of } \\
m\end{array}$} \\
\hline & 988 & 1019 & 1051 & 1083 & \\
\hline 1 & 0.98 & $0.98-1.12$ & 0.98 & $1.01-1.10$ & 1.05 \\
\hline 2 & - & - & - & $0.98-1.03$ & 1.0 \\
\hline 3 & 1.0 & 0.93 & 0.88 & $0.90-1.0$ & 0.96 \\
\hline 4 & 0.99 & 1.01 & 0.91 & 0.96 & 0.97 \\
\hline 5 & 0.92 & 0.94 & 0.95 & $0.89-0.90$ & 0.93 \\
\hline 6 & 0.86 & 0.94 & 0.93 & 0.94 & 0.92 \\
\hline 7 & 0.90 & 0.88 & 0.87 & 0.80 & 0.86 \\
\hline 8 & $0.88-0.92$ & 0.90 & 0.93 & - & 0.90 \\
\hline 9 & 0.77 & 0.82 & 0.89 & $0.75-0.86$ & 0.82 \\
\hline
\end{tabular}

reactant may now be evaluated by the slopes of straight lines depicted in Fig. 4. The value of $m$ evaluated for each sample is listed in Table 2. As already mentioned, the order $m$ is dependent only on the kinds of sample used and is independent of reaction conditions. The values of $m$ are plotted against the corresponding standard deviation $\sigma$ of grains constituting pellets in Fig. 3. All the data agree well with the theoretical prediction depicted as a solid line in the figure. One may conclude from Figs. 3 and 6 that the oxidation of the present zinc sulfide pellets follows the reaction kinetics derived in the preceding chapter.

\subsection{Rate constant}

On confirming the kinetics, the surface rate constant may now be evaluated by observed initial conversion rate data on the basis of Eq. (11). Figure 7 illustrates the temperature dependency of the rate constant. This Arrhenius plot provides the activation energy of oxidation reaction as $244 \mathrm{~kJ} / \mathrm{mol}$. This value is very close to $252 \mathrm{~kJ} / \mathrm{mol}$ observed by Ong et al. ${ }^{12}$ ) $210 \mathrm{~kJ} / \mathrm{mol}$ by Cannon and Denbigh, ${ }^{2)}$ and $261 \mathrm{~kJ} /$ mol by Gerlach and Stichel. ${ }^{5)}$

Cannon and Denbigh ${ }^{2)}$ and Ong et al. ${ }^{12)}$ measured the oxidation rate of sphalerite single crystals of a few $\mathrm{mm}$ in size. They found that the reaction proceeded forming an unreacted core in the crystal in the regime of chemical reaction control. In the experiments by Cannon and Denbigh the rate of oxidation was also found to be proportional to the square root of the oxygen partial pressure. These facts strictly match the present kinetics although the size of crystals in which the reaction takes place is significantly different. The surface rate constant evaluated by Cannon and Denbigh is shown in Fig. 7 to be in good agreement with the present results. The rate constant reproduced from the data by Ong et al. by approximating $n=1 / 2$ also agrees fairly well with the present results.

The surface rate constant $k^{\prime}$ may be correlated in the temperature range $953-1113 \mathrm{~K}$ by

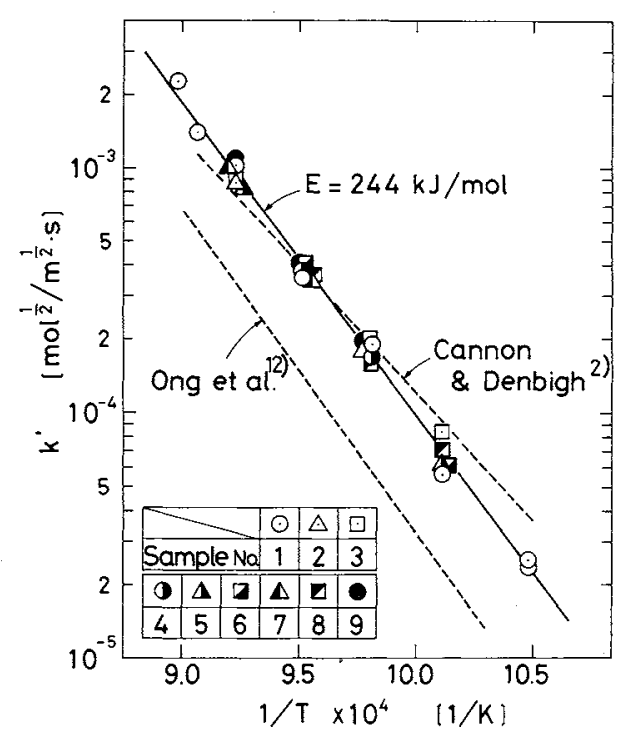

Fig. 7. Arrhenius plot of surface rate constant $k^{\prime}$ in comparison with results by other workers.

$$
k^{\prime}=5.56 \times 10^{8} \mathrm{e}^{-244 / R T} \quad\left(\mathrm{~mol}^{1 / 2} / \mathrm{m}^{1 / 2} \cdot \mathrm{s}\right)
$$

\section{Discussion}

A number of experimental works on the oxidation of zinc sulfide have been carried out on single-particle systems, including both single crystals and grainy porous pellets. The rate data by individual workers are often compared on the basis of initial reaction rate per unit external surface area of the particle. ${ }^{4,6,11,14)}$ This comparison provides a significant difference in the data, especially showing the rate values for single crystals to be very much smaller than those for grainy porous pellets.

Since the reaction in nonporous single crystals was revealed to proceed on the reacting interface in the regime of chemical reaction control, ${ }^{2,12)}$ the reaction rate constant evaluated on this basis may be acceptable for single-crystal systems. However, in view of the zone-type reaction occurring in a porous particle, ${ }^{13,14)}$ the interfacial reaction model is not applicable to reacting grainy porous pellets. Thus, the rate values evaluated on the basis of external surface area may become extremely large due to the contribution of internal surface area in the reacting zone.

Limiting the situation in the regime where the conversion progresses under chemical reaction control, the reaction was often observed to proceed homogeneously throughout the pellet. ${ }^{3,17)}$ Takamura et al. ${ }^{17)}$ has shown that the oxidation reaction of grainy porous zinc sulfide pellets follows a rate equation represented by

$$
\frac{d \bar{X}_{B}}{d t}=\left(\frac{d \bar{X}_{B}}{d t}\right)_{t=0} \cdot\left(1-\bar{X}_{B}\right)
$$

Equation (13) corresponds to a special case of Eq. 


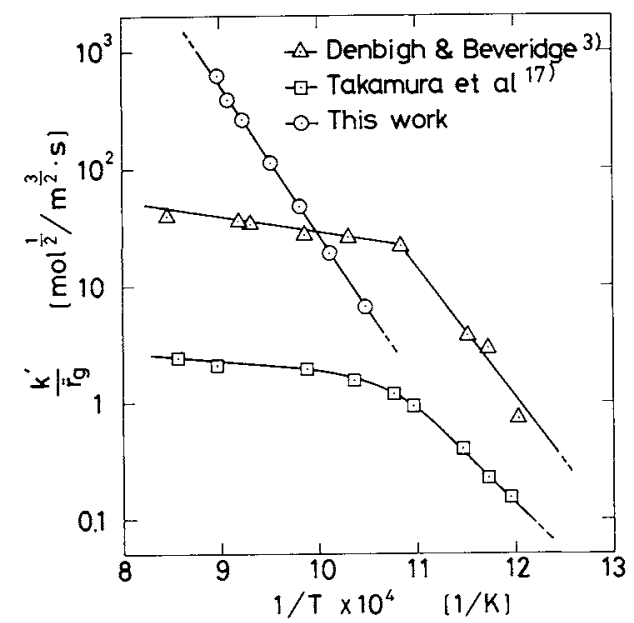

Fig. 8. Surface rate constant evaluated from literature data on oxidation of porous pelleted zinc sulfide.

(10) for $m=1$. Assuming that the zinc sulfide grains constituting their pellets also have the log-normal size distribution which most feequently applies, ${ }^{1,10,15)}$ the standard deviation may be estimated to be about 0.5 from Fig. 3. Thus, their data on the initial reaction rate may be subjected to analysis by Eq. (11) to evaluate the rate constant.

Since no information about grain radius is given in the literature, the rate constant may be evaluated in the following form.

$$
\frac{k^{\prime}}{\overline{\bar{r}}_{g}}=\frac{\mathrm{e}^{-\sigma^{2} / 2}}{3 b v_{B} C_{A}{ }^{n}}\left(\frac{d \bar{X}_{B}}{d t}\right)_{t=0}
$$

Figure 8 illustrates the rate constant reproduced with $\sigma=0.5$ and $n=0.5$ from the data by Takamura et al. In this figure the rate constant estimated from the data by Denbigh and Beveridge, ${ }^{3)}$ assuming the same values for $\sigma$ and $n$, is also shown.

The straight portions at lower temperature, terminating at around $850 \mathrm{~K}$, are considered to represent the chemical reaction control for the process. In the higher-temperature region the data seem to be influenced by diffusion limitation.

The rate constants by these workers are located close to the results of this work. The discrepancy between them is considered to be due to the differences in the properties of sulfides and also in the mean grain radius appearing in the denominator of the ordinate. The activation energies are evaluated to be $160 \mathrm{~kJ} / \mathrm{mol}$ for the data by Takamura et al. and $230 \mathrm{~kJ} / \mathrm{mol}$ by Denbigh and Beveridge, respectively. These values are not very different from the $244 \mathrm{~kJ} /$ mol obtained in this work.

It is already shown in Fig. 7 that the magnitude of the rate constant in this work agrees well with that for the oxidation of sphalerite single crystals. Therefore, the above interpretation reveals that the surface rate constants evaluated in the oxidation of porous pellets do not differ very much from those of crystals but are of the same order of magnitude.

\section{Conclusion}

Oxidation of porous zinc sulfide pellets, composed of fine grains which are nonuniform in size, was carried out using a differential flow reactor. On confirming that the grain size of the zinc sulfide powder is log-normally distributed, the rate data were analyzed by the grain model accounting for grain size distribution.

The overall conversion rate was represented by a simple power law rate expression. The reaction order with respect to the solid reactant varied with the standard deviation of the grain size distribution. This variation was in good agreement with the theoretical prediction by the grain model accounting for grain size distribution. The reaction order with respect to the gaseous reactant yielded $1 / 2$.

The surface rate constant was found to be correlated in an Arrhenius equation. The results obtained agreed well with those for the oxidation of sphalerite single crystals reported by other workers. The rate data in the literature on the oxidation of porous pelleted zinc sulfide were also subjected to interpretation by the present kinetic model. The surface rate constant reproduced from the literature data also agreed well in order of magnitude with the data from this work.

\section{Acknowledgment}

The authors are grateful to Dr. Jusuke Hidaka, Lecturer of Dept. of Chemical Engineering, Doshisha University, Kyoto, for his helpful advice on classification of fine powder by microsieves. The authors are also indebted to Dr. Susumu Namba, Professor of Dept. of Electrical Engineering, Osaka University, Osaka, for every facility given to them for taking SEM pictures.

\begin{tabular}{|c|c|c|}
\hline \multicolumn{3}{|c|}{ Nomenclature } \\
\hline$b$ & $=$ & stoichiometric coefficient \\
\hline$C_{A}$ & $=$ & gaseous reactant concentration \\
\hline$E$ & $=$ & activation energy \\
\hline$f(\tau)$ & & $\begin{array}{l}\text { probability density function for complete } \\
\text { conversion time represented by log-normal } \\
\text { distribution }\end{array}$ \\
\hline$g\left(r_{g}\right)$ & $=$ & $\begin{array}{l}\text { probability density function for grain radius } \\
\text { represented by log-normal distribution }\end{array}$ \\
\hline$k^{\prime}$ & $=$ & surface rate constant \\
\hline$m$ & $=$ & $\begin{array}{l}\text { reaction order with respect to unconverted } \\
\text { fraction of solid }\end{array}$ \\
\hline$n$ & $=$ & $\begin{array}{l}\text { reaction order with respect to gaseous } \\
\text { reactant }\end{array}$ \\
\hline$R$ & $=$ & ideal gas law constant \\
\hline$r_{g}$ & $=$ & grain radius \\
\hline $\bar{r}_{g}$ & $=$ & mean grain radius \\
\hline$T$ & $=$ & absolute temperature \\
\hline$t$ & $=$ & reaction time \\
\hline$v_{B}$ & $=$ & molar volume of $B$ \\
\hline$X_{B}$ & $=$ & extent of conversion of a grain \\
\hline $\bar{X}_{B}$ & $=$ & average extent of conversion of a pellet \\
\hline
\end{tabular}




$$
\begin{array}{rlr}
= & \text { a parameter defined by } \xi=\ln (\tau / \bar{\tau}) / \sigma & {[-]} \\
= & \text { standard deviation of grain size distribution } \\
= & \text { complete conversion time for a grain with } \\
& \text { radius } r_{g} & \\
= & \text { mean of complete conversion time of grains }
\end{array}
$$

\title{
Literature Cited
}

1) Bartlett, R. W., N. G. Krishnan and M. C. van Hecke: Chem. Eng. Sci., 28, 2179 (1973).

2) Cannon, K. J. and K. G. Denbigh: Chem. Eng. Sci., 6, 145 (1957).

3) Denbigh, K. G. and G. S. G. Béveridge: Trans. Instn. Chem. Engrs, 40, 23 (1962)

4) Fukunaka, Y., T. Monta, Z. Asaki and Y. Kondo: Metal. Trans., 7B, 307 (1976).

5) Gerlach, J. and W. Stichel: Z. Erz. Metal., 17, 427 (1964); Chem. Abstr., 63, 13930h (1964).

6) Gray, N. B., M. R. Harvey and G. M. Wills: Proc. Richardson Conf. Phys. Chem. Process Metallurgy, Imperial College, London (1973).

7) Kimura, S., S. Tone and T. Otake: J. Chem. Eng. Japan, 15, 73 (1982).

8) Kimura, S., S. Tone and T. Otake: J. Chem. Eng. Japan, 14, 491 (1981).

9) Levenspiel, O.: "Chemical Reaction Engineering," 2nd ed., John Wiley, New York (1972).

10) Mcllvried, H. G. and F. E. Massoth: Ind. Eng. Chem., Fundam., 12, 225 (1973).

11) Natesan, K. and W. O. Philbrook: Trans. Metal. Soc. AIME, 245, 2243 (1969).

12) Ong, J. N., M. E. Wadsworth and M. W. Fassell: Trans. Metal. Soc. AIME, 206, 257 (1956).

13) Prasannan, P. C. and L. K. Doraiswamy: Chem. Eng. Sci., 37, 925 (1982).

14) Rao, T. R. and R. Kumar: Chem. Eng. Sci., 37, 987 (1982).

15) Szekely, J. and M. Propster: Chem. Eng. Sci., 30, 1049 (1975).

16) Sohn, H. Y. and J. Szekely: Chem. Eng. Sci., 27, 763 (1972).

17) Takamura, T., K. Yoshida and D. Kunii: J. Chem. Eng. Japan, 7, 276 (1974)

18) Wen, C. Y. and M. Ishida: Environ. Sci. Technol., 7, 703 (1973).

(Presented at the 16th Autumn Meeting of The Society of Chemical Engineers, Japan.)

\section{HYDROLYSIS OF DIETHYL SUCCINATE CATALYZED BY CATION EXCHANGE RESIN}

\author{
Motonobu GOTO, SHIgeo GOTO AND the late Hideo TESHIMA \\ Department of Chemical Engineering, Nagoya University, Nagoya 464
}

\begin{abstract}
Hydrolysis of diethyl succinate as a consecutive reaction was investigated in a batch slurry reactor catalysed by cation exchange resin.

Two models, one realistic and one simplified, were derived. The differences between the models were not significant under the conditions of this study, and both showed good agreement with experimental data.

The catalytic activities for gel-type resins were higher than for a macroreticular (MR)-type resin and a free acid. For gel-type resins, the reaction rate per equivalent increased as crosslinking decreased.
\end{abstract}

\section{Introduction}

Cation exchange resin of $\mathrm{H}^{+}$form has been used as acid catalyst for liquid-phase reaction and it has several advantages over free acid, such as easy separation from products. Since reactants, however, must reach active sites in the resin, the reaction rate may be influenced by particle size and crosslinking.

Many investigations have been made of the hydrolysis of ester catalyzed by cation exchange resin. The catalytic effectiveness factor was introduced to analyze intraparticle diffusion. ${ }^{14,15)}$

Recently, a micro-macro pore model has been proposed for macroreticular resin catalyst. ${ }^{7)}$ It was

\footnotetext{
Received August 13, 1982. Correspondence concerning this article should be addressed to $\mathrm{S}$. Goto.
}

reported $^{16,18)}$ that the activity of resin catalyst was greater than that of free-acid catalyst.

Although these studies were limited to simple reactions, many industrial reactions are complex and selectivity is an important factor along with catalyst activity. Theoretical studies of the catalytic effectiveness factor and selectivity for consecutive reactions have been made in terms of a single-pore model,,$^{19,20}$ ) macro-micro pore model $^{3)}$ and variable diffusivity model. ${ }^{2)}$ Experimental studies of common solid catalysts have been reported on the hydrogen of acetylene, ${ }^{10)}$ of mesityle oxide ${ }^{5)}$ and of phenylacetylene. ${ }^{9)}$

In this study, hydrolysis of diethyl succinate catalyzed by cation exchange resin of both gel and macroreticular (MR) types was investigated in a batch slurry reactor. Two models, one realistic and 